\title{
The Swift-XRT observations of HBL Source 1ES $1959+650$
}

\section{Bidzina Kapanadze ${ }^{* a b}$, Patrizia Romano ${ }^{b}$, Stefano Vercellone ${ }^{b}$, Sergo Kapanadze ${ }^{a}$, Grigol Kharshiladze ${ }^{c}$}

${ }^{a}$ E. Kharadze Abastumani Astrophysical Observatory, Ilia State University, Colokashvili Av. 3/5, Tbilisi, Georgia, 0162

${ }^{b}$ INAF, Istituto di Astrofisica Spaziale e Fisica Cosmica, Via U. La Malfa 153, Palermo, Italy, I-90146

${ }^{c}$ Georgian Technical University, Kostava 77, Tbilisi, Georgia, 0175

E-mail: bidzina_kapanadzedilauni.edu.ge, romanodifc.inaf.it.

stefanodifc.inaf.it sergo_kapanadze.1@ilauni.edu.ge:

kharshiladze72@mail.ru

$1 \mathrm{ES} 1959+650$ is one of the X-ray bright blazars with the highest and mean $0.3-10 \mathrm{keV}$ count rates of $13.74 \mathrm{cts} \mathrm{s}^{-1}$ and $6.8 \mathrm{cts} \mathrm{s}^{-1}$, respectively, derived from the Swift-XRT observations performed since 2005 April. During this period, the source showed several longer-term flares by a factor of 2-6 and timescales of 30-130 d which sometimes were not accompanied with an increasing activity in the $\gamma$-ray or lower-energy parts of the spectrum and vice versa. The 18 cases of intra-day flux changes with timescales down to $0.6 \mathrm{ks}$ are revealed, generally observed in the higher brightness states. The spectra were mostly curved with broad ranges of photon index, curvature parameter, hardness ratio and synchrotron SED peak location. The distribution of the curvature parameter shows that the emitting particles in 1ES 1959+650 should undergo an effective stochastic acceleration at the shock front, similar to other TeV-detected HBLs.

Swift: 10 Years of Discovery,

2-5 December 2014

La Sapienza University, Rome, Italy

\footnotetext{
* Speaker.
} 


\section{Introduction}

BL Lacertae objects (BLLs) represent one of the most extreme AGN subclasses with quasifeatureless spectra, high optical and radio polarization, compact radio-morphology, strong flux variability and broad continuum extending from radio to very high energy $\gamma$-rays, explained as a result from a relativistically boosted non-thermal emission radiated by the jet closely aligned to the line-of-sight [1].

1ES 1959+650 is a high-energy peaked BL Lacertae source (HBL, i.e. a BL Lac object with a synchrotron peak in the UV-X-ray part of the spectrum [2]) with $z=0.048, \mathrm{TeV}$-detected and hosted by a bright elliptical galaxy (see [3]). It has been Observed 120 times by X-ray Telescope (XRT) onboard the Swift satellite since 2005 April 19 with a total exposure time of $174 \mathrm{ks}$. Our contribution is based on the preliminary results obtained from the analysis of these observations.
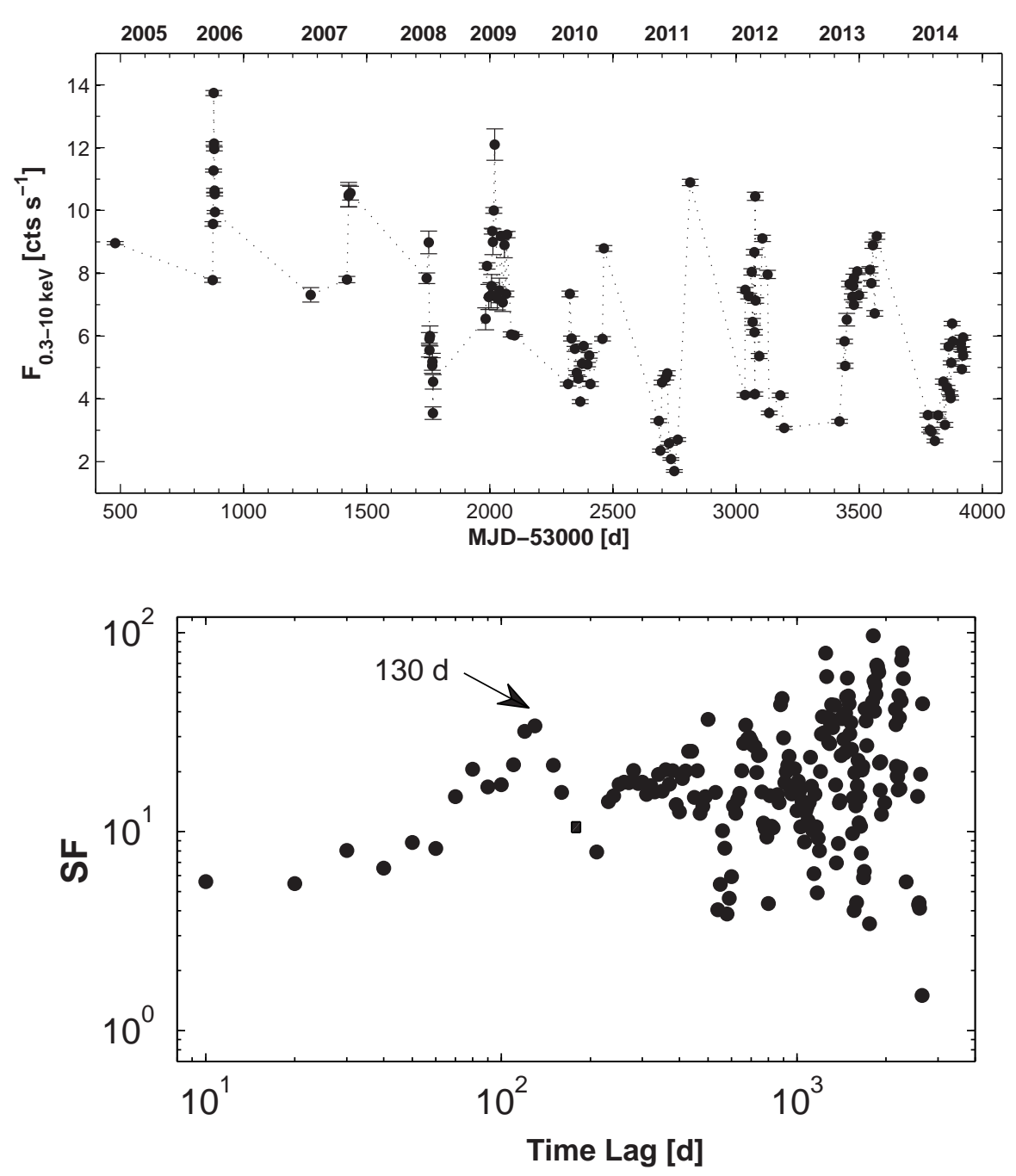

Figure 1: Historical 0.3-10 keV light curve of 1ES 1959+650 and corresponding structure function. 


\section{Long-term X-ray Flux Variability}

The source was highly variable with the maximum-to-minimum flux ratio of 8 , and showed flares by a factor of 2-6 on the weeks-to-months timescales (see Fig. 1, upper panel). The flux variability showed an erratic character changing its amplitude and minimum flux level during the flares occurring in different epochs. During the $9.5 \mathrm{yr}$ period, the highest and mean fluxes were $13.74 \mathrm{cts} \mathrm{s}^{-1}$ and $6.8 \mathrm{cts} \mathrm{s}^{-1}$, respectively. Therefore, 1ES $1959+650$ is one of the brightest blazars in the X-rays with unabsorbed $0.3-10 \mathrm{keV}$ fluxes of $5.76 \times 10^{-11}-5.39 \times 10^{-10} \mathrm{erg} \mathrm{cm}^{-2} \mathrm{~s}^{-1}$.
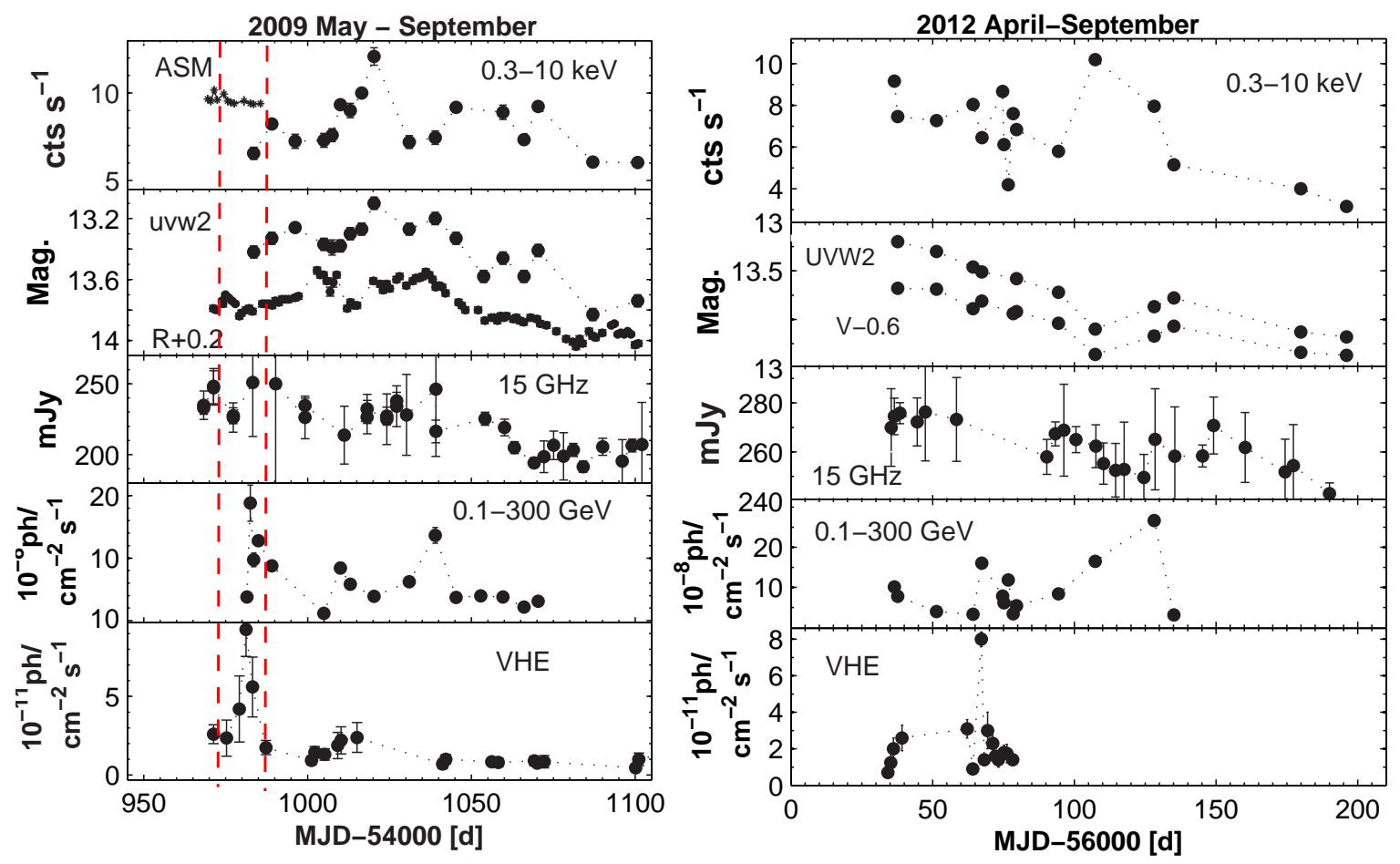

Figure 2: Multiwavelength variability of 1ES 1959+650 in different epochs. The epochs with uncorrelated variabilities in different spectral bands are shown between the vertical dashed lines.

The initial increasing part of the structure function (SF) plot, constructed via the $10 \mathrm{~d}$ bins (Fig. 1, lower panel), exhibits breaks at the time lags 30-130 d, which can be considered as variability timescales in different epochs.

Sometimes, the X-ray flares were not accompanied by an increasing activity in other spectral bands and vice versa. The most remarkable event was a VHE flare by a factor of 4 at the end of 2009 May, accompanied by that in the $0.1-300 \mathrm{GeV}$ band. However, we do not observe a similar behaviour in the synchrotron part of the spectrum in this period. From the past observations, the source showed an "orphan"VHE flare on 2002 June 4 (see [4]).

The source was in a high X-ray state during 2012 April-July when it showed small-amplitude fluctuations around the $7 \mathrm{cts} \mathrm{s}^{-1}$ level. In the epoch of the highest X-ray flux (accompanied by an outburst also in the 0.1-300 GeV energy bands), the source showed its lowest brightness states in the radio-UV parts of the spectrum. 


\section{Intra-day X-ray Flux Variability}

For this source, the 18 cases of intra-day flux variability (IDV) at the $99.9 \%$ confidence with fractional amplitudes of 3.2-16.7\% and timescales ranging from $25 \mathrm{ks}$ down to about $0.6 \mathrm{ks}$ are revealed, generally observed during the higher brightness states.

In the upper panel of Fig. 3, we present an example of the IDV observed on 2006 May 23 when the source showed two successive increases in the $0.3-10 \mathrm{keV}$ count rate by $10-15$ per cent. Along with the longer-timescale variability $\left(t_{\mathrm{var}} \backsim 5 \mathrm{ks}\right)$, we have found a very fast event with $t_{\mathrm{var}} \approx 600 \mathrm{~s}$.

Another five cases of X-ray IDVs at the $99.5 \%$ confidence (so-called possible variabilities) are also revealed. The second row of Fig. 3 presents the two examples of these events. The source showed a decay by the fractional amplitude $F_{\mathrm{var}}=6 \%$ during $840 \mathrm{~s}$ without a clear timescale during the first orbit of the 2009 August 23 pointing. The two successive fluctuations with $t_{\mathrm{var}} \approx 250 \mathrm{~s}$ and $F_{\text {var }}=4.75 \%$ were observed on 2013 July 3.
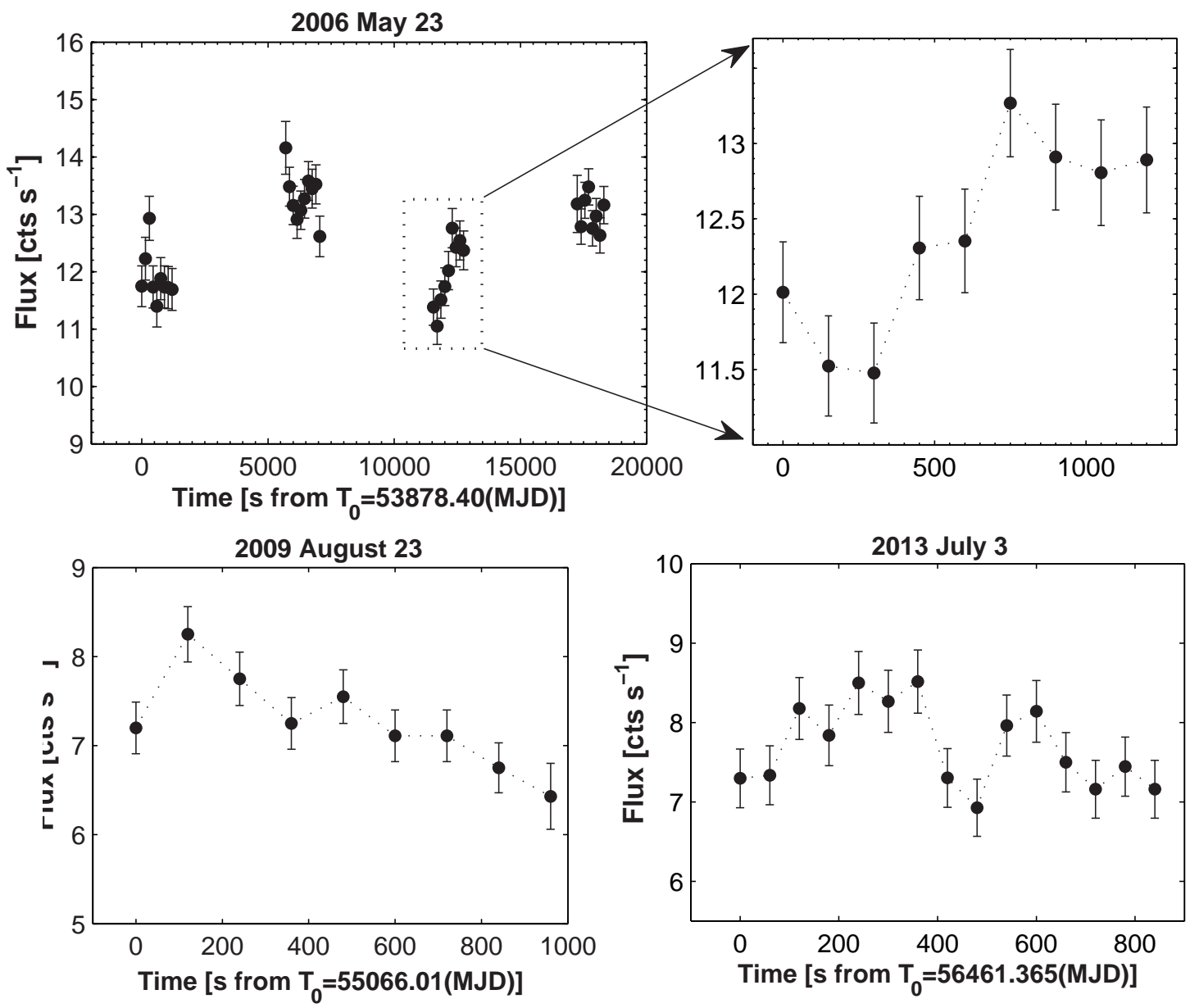

Figure 3: Examples of Intra-day X-ray flux variability at different confidence levels. 


\section{Spectral analysis}

The 0.3-10 keV spectra are generally fitted well with the logparabolic law [5]

$$
F(E)=K\left(E / E_{1}\right)^{\left(-a+b l o g\left(E / E_{1}\right)\right.} \quad \mathrm{ph} \mathrm{cm}^{-2} \mathrm{~s}^{-1} \mathrm{keV}^{-1}
$$

with the reference energy $E_{1}$ fixed to $1 \mathrm{keV} ; a$ - the photon index at the energy $E_{1} ; b$ - the curvature parameter; $K$ - the norm. The values of these parameters are derived during the fit process. The location of SED peak is given by

$$
E_{\mathrm{p}}=E_{1} 10^{(2-a) / 2 b}
$$

For the equivalent hydrogen column density

$$
N_{\mathrm{H}}=1.00 \times 10^{21} \mathrm{~cm}^{-2},
$$

(fixed to the Galactic value obtained from the LAB Survey), we get

$$
\begin{gathered}
a=1.76-2.39 \\
b=0.14-0.83 \\
E p=0.12-1.84 k e V
\end{gathered}
$$

These parameters generally showed a variability with X-ray flux. The spectral changes generally followed a "harder-when-brighter"evolution of the source with pure clockwise $(\mathrm{CW})$ or dominantly CW loops in the hardness ratio-0.3-10 keV flux plane (see Fig. 4).
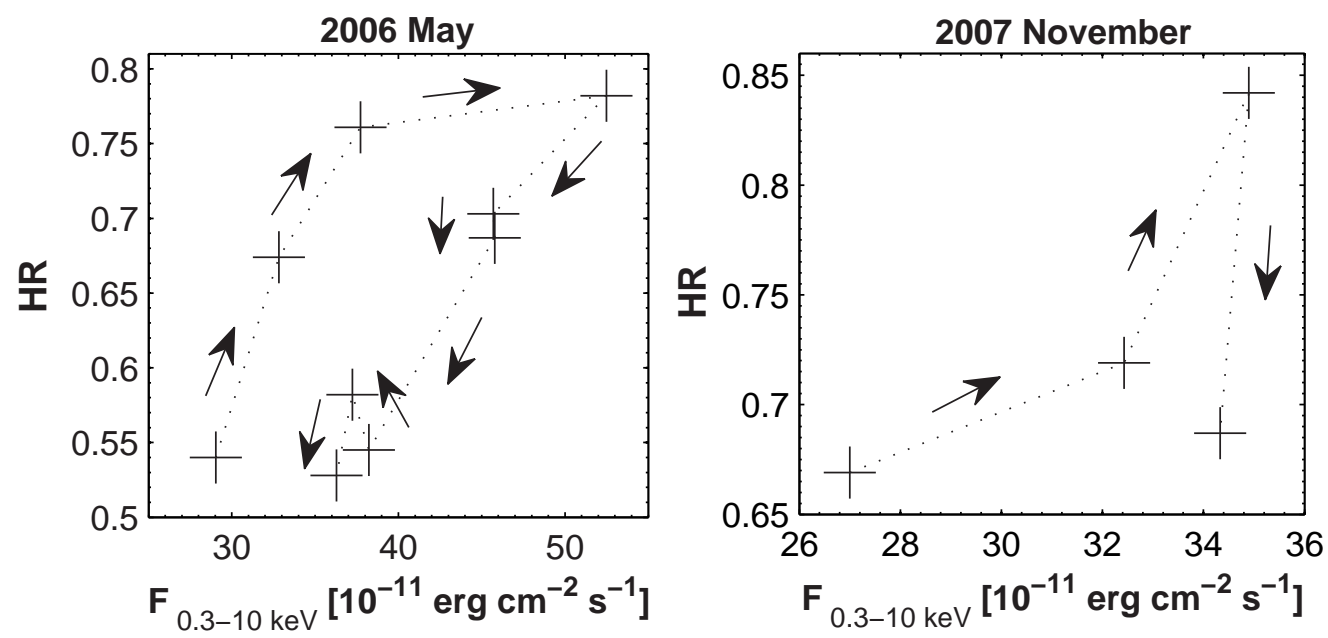

Figure 4: Spectral hysteresis in different epochs.

\section{Discussion}

- One-zone SSC model was not always suitable for this HBL - we revealed "orphan"X-ray and $\gamma$-ray flares. Similar events were also reported in the previous studies of 1ES1959+650. These events may be related to the existence of separate populations of accelerated electrons in the source. 
- A curved spectral distribution of the particles with energy develops when the acceleration probability is a decreasing function of the electron energy (so-called energy-dependent acceleration probability process (EDAP [5]).

- In this scenario, a linear relation between the spectral parameters $a$ and $b$ is expected. However, we have not found a significant correlation between them for any flare. Therefore, EDAP should be less effective for our target.

- A curved spectral distribution can be also established via the stochastic acceleration which arises from the magnetic turbulence close to the shock front [6]. [7] showed that the electrons should undergo an efficient stochastic acceleration when the synchrotron SEDs are relatively broad $(b \sim 0.3)$ while they are narrower $(b \sim 0.7)$ in the opposite case.

- Based on the distribution of curvature parameter peaking at $b=0.39$, and the observed anticorrelation between the curvature parameter and the 0.3 UU10 keV flux, the most likely mechanism responsible for producing $\mathrm{X}$-ray emission during the flares is the stochastic acceleration of the electrons.

\section{Acknowledgements}

B.K. thanks Shota Rustaveli National Science Foundation for the travel grant N03/116 and Fundamental Research Grant FR/377/6-290/14. He is very grateful to Neil Gehrels for approving his ToO requests. P.R. acknowledges the contract ASI-INAFI/004/11/0. This research has made use of XRTDAS and the data from the OVRO 40m telescope monitoring program. We acknowledge the helpful service of HEASARC.

\section{References}

[1] A. Abdo et al., THE SPECTRAL ENERGY DISTRIBUTION OF FERMI BRIGHT BLAZARS, ApJ,716, $30(2010)$

[2] P. Padovani \& P. Giommi, THE CONNECTION BETWEEN X-RAY- AND RADIO-SELECTED BL LACERTAE OBJECTS, ApJ, 444, 567 (1995)

[3] B. Kapanadze \& E. Janiashvili, Variable synchrotron emission from BL Lacertae objects. II. Optical and X-ray flares in HBL source IES 1959+650, Ap\&SS, 339, 339 (2012)

[4] H. Krawczynski et al., MULTIWAVELENGTH OBSERVATIONS OF STRONG FLARES FROM THE TeV BLAZAR 1ES 1959+650, ApJ, 601, 151 (2004)

[5] E. Massaro et al., Log-parabolic spectra and particle acceleration in the BL Lac object Mkn 421: Spectral analysis of the complete BeppoSAX wide band X-ray data set, A\&A, 413,489 (2004)

[6] A. Tramacere et al., Swift observations of the very intense flaring activity of Mrk 421 during 2006. I. Phenomenological picture of electron acceleration and predictions for MeV/GeV emission, A\&A, 501, 879 (2009)

[7] F. Massaro et al., X-RAY AND TeV EMISSIONS FROM HIGH-FREQUENCY-PEAKED BL LAC OBJECTS, ApJ, 742, L32 (2011) 